\title{
Natural Course and Factors Associated with Allergic Diseases in Early Life: Data from a Developing Asian Country
}

\author{
Thansinee Saetae ${ }^{1}$, Pantipa Chatchatee ${ }^{1}$, Yong Poovorawan ${ }^{1}$, Nasamon Wanlapakorn ${ }^{1}$, \\ Kanlada Wongworapat ${ }^{1}$, Surasith Chaithongwongwattana ${ }^{1}$, Jarungchit Ngamphaiboon ${ }^{1}$, \\ Leuridan Elke ${ }^{2}$, and Narissara Suratannon ${ }^{1}$ \\ ${ }^{1}$ Chulalongkorn University Faculty of Medicine \\ ${ }^{2}$ University of Antwerp Drie Eiken Campus
}

November 20, 2020

\begin{abstract}
Background: The incidence of allergic diseases has been rising in the last decades. The interplay between genetic and environmental factors underlined these complex diseases. Data concerning natural history and risk factors associated with allergic diseases in developing Asian countries are limited. Methods: A population-based birth cohort study was conducted in Bangkok, Thailand. Perinatal and postnatal factors were prospectively collected by questionnaires. Sensitization was identified by skin prick test. Food allergy (FA) was confirmed by oral food challenge. Results: A total of 369 mother-children pairs were enrolled and follow-up until the age of 30 months. The cumulative incidence of allergic diseases was $46.2 \%$. High family income significantly increased risk of atopic dermatitis (AD) and ever-wheezing. Maternal history of allergic diseases and exclusive breastfeeding longer than six months significantly increased risk of FA. Antibiotics use in infants significantly associated with FA, ever-wheezing and rhinitis. Infants with FA had a 6.5-fold increased risk of AD while infants with AD had an 8.5-fold increased risk of FA. AR had a 3.5-fold increased risk in developing ever-wheezing. One-third of the subjects had aeroallergen sensitization. Conclusion: Prevalence of allergic diseases in developing Asian country was considerably high. AD and FA were the most prevalent allergic diseases during the first years of life. Meanwhile, the prevalence of wheezing and rhinitis subsequently increased over time. High sensitization rate to aeroallergens was found in the first two years of life. Maternal atopy, high family income, early life exposure to antibiotics and prolonged breast-feeding increased risk of allergic diseases in our cohort.
\end{abstract}

\section{Natural Course and Factors Associated with Allergic Diseases in Early Life: Data from a Developing Asian Country}

Thansinee Saetae ${ }^{1^{*}}$, Pantipa Chatchatee ${ }^{1 *}$, Yong Poovorawan ${ }^{2}$, Nasamon Wanlapakorn ${ }^{2}$, Kanlada Wongworapat ${ }^{1}$,Surasith Chaithongwongwatthana ${ }^{3}$, Jarungchit Ngamphaiboon ${ }^{1}$, Elke Leuridan ${ }^{4}$, Narissara Suratannon $^{1 * *}$

${ }^{1}$ Pediatric Allergy and Clinical Immunology Research Unit, Division of Allergy and Immunology, Department of Pediatrics, Faculty of Medicine, Chulalongkorn University, King Chulalongkorn Memorial Hospital, The Thai Red Cross Society, Bangkok, Thailand ${ }^{2}$ Center of Excellence Clinical Virology, Faculty of Medicine, Chulalongkorn University, Bangkok, Thailand, ${ }^{3}$ Department of Obstetrics and Gynecology, Faculty of Medicince, Chulalongkorn University, Bangkok, Thailand, ${ }^{4}$ Centre for the Evaluation of Vaccination, Vaccine and Infectious Diseases Institute, University of Antwerp-Campus Drie Eiken, Antwerp, Belgium

* These authors contributed equally to this work

A running title: Progression of Allergy in Developing Countries

\section{** Corresponding author:}


Narissara Suratannon

Pediatric Allergy and Clinical Immunology Research Unit, Division of Allergy and Immunology, Department of Pediatrics, Faculty of Medicine, Chulalongkorn University, King Chulalongkorn Memorial Hospital, The Thai Red Cross Society

1873 Rama IV Rd, Pathum Wan, Bangkok 10330, Thailand

E-mail: narissara.su@chula.ac.th

Word count: 2498 words; 2 Figures; 4 Tables

Material in the electronic repository: additional Methods section

Conflict of Interest: All authors have no relevant financial relationships to disclose.

Financial support : This study was supported by Ratchadapisek Research Funds (Grant No. CU-GR(S)_61_38_30_03), Chulalongkorn University, Bankgok, Thailand and Thrasher Research Fund.

\section{Abstract}

Background: The incidence of allergic diseases has been rising in the last decades. The interplay between genetic and environmental factors underlined these complex diseases. Data concerning natural history and risk factors associated with allergic diseases in developing Asian countries are limited.

Methods: A population-based birth cohort study was conducted in Bangkok, Thailand. Perinatal and postnatal factors were prospectively collected by questionnaires. Sensitization was identified by skin prick test. Food allergy (FA) was confirmed by oral food challenge.

Results: A total of 369 mother-children pairs were enrolled and follow-up until the age of 30 months. The cumulative incidence of allergic diseases was $46.2 \%$. High family income significantly increased risk of atopic dermatitis $(\mathrm{AD})$ and ever-wheezing. Maternal history of allergic diseases and exclusive breastfeeding longer than six months significantly increased risk of FA. Antibiotics use in infants significantly associated with FA, ever-wheezing and rhinitis. Infants with FA had a 6.5-fold increased risk of AD while infants with AD had an 8.5-fold increased risk of FA. AR had a 3.5-fold increased risk in developing ever-wheezing. One-third of the subjects had aeroallergen sensitization.

Conclusion: Prevalence of allergic diseases in developing Asian country was considerably high. AD and FA were the most prevalent allergic diseases during the first years of life. Meanwhile, the prevalence of wheezing and rhinitis subsequently increased over time. High sensitization rate to aeroallergens was found in the first two years of life. Maternal atopy, high family income, early life exposure to antibiotics and prolonged breast-feeding increased risk of allergic diseases in our cohort.

Keywords: natural history; risk factor; atopic dermatitis; food hypersensitivity; asthma; rhinitis, allergic; Asia, Southeastern; cohort studies

Impact statement: This is one of only few birth cohort conducting in developing Asian countries. Our study contributes to deeper insights in similarities and differences of natural history and risk factors in early life allergic manifestations among different population groups.

\section{Introduction}

Allergic diseases have been increasing in the last decades, particularly in developing countries ${ }^{1}$. This increase is likely a consequence of environmental factors that heightens the risk of allergic diseases in genetically susceptible individuals $^{2}$. To date, allergy birth cohort studies are from western countries ${ }^{3,4}$ and modern Asian countries ${ }^{5-8}$. The generalizability of these results to the context of developing Asian countries may not be relevant since the environment, feeding patterns and lifestyles are largely different.

Positive family history of atopy is one of the important predisposing factors of allergic diseases ${ }^{9,10}$. Maternal atopy is associated with allergic diseases than paternal influence ${ }^{11}$. The protective effects of breast feeding 
against allergy remain a debating issue ${ }^{12}$. Environmental factors related to hygiene hypothesis theory, such as high socioeconomic status, extended family, antibiotics exposure during infancy period, farm environment and household furry pets, have been proposed to be associated with allergic diseases ${ }^{13}$. The rationale behind this theory is that families with hygienic lifestyles are less exposed to microorganisms. So the immature immune system of the young infants might be driven towards allergic inflammation.

The atopic march refers to the progression of allergic diseases during infancy and childhood ${ }^{14}$. Classically, the atopic march begins with atopic dermatitis (AD) or food allergy (FA) then progresses to asthma or allergic rhinitis (AR) ${ }^{14}$. The co-occurrence between the diseases are also common ${ }^{15,16}$. These data support the existence of a shared set of polymorphisms in allergic patients. However, previous literature has shown that only one-third to half of the subjects with $\mathrm{AD}$ and FA developed atopic march later in life ${ }^{17}$. Besides, not every subject with asthma and AR preceded with $\mathrm{AD}$ or $\mathrm{FA}^{17}$.

Using a population-based birth cohort manner, our study aimed to describe the incidence, and natural course including of atopic march and to identify factors associated with allergic diseases in Thai population, which reflects developing Asian countries.

\section{Methods}

This study is a population-based birth cohort study conducted during July 2015 and July 2019 at King Chulalongkorn Memorial Hospital (KCMH), in Bangkok, Thailand. Pregnant women with 27th to 36th week of gestation who was living in Bangkok and visited KCMH between July 2015 to January 2016 were invited to join our study. The inclusion criteria were healthy full-term infants who were born from low risk healthy pregnant women. Infants born from multiple pregnancies or high-risk pregnant women, preterm infants and infants with congenital anomalies were excluded. Informed consent was obtained from all parents. Overall, 600 women were screened, 500 mother-children pairs were eligible to the inclusion criteria in which 369 pairs were enrolled. We performed longitudinal assessments of the subjects from the third trimester of pregnancy until the child reached 30 months of age. The study was performed according to the Helsinki Guidelines and approved by the Ethics Committee of KCMH (IRB No. 358/58). Details of clinical data collection, definition of allergic diseases, methods to define sensitization to food allergens and aeroallergens were described in the Appendix.

\section{Statistical analysis}

The statistical analysis was performed by IBM SPSS Statistics for Windows, version 22 (IBM Corp., Armonk, N.Y., USA). Characteristics of the patients were presented using the median (range) for continuous data or the number and percentage for categorical data. Associations between factors including aeroallergen sensitization and allergic diseases were estimated using univariate and multivariable logistic regression which represented in odds ratio and 95\% confidence intervals (CI). Cox proportional hazard regression was used to find factors associated with outgrown of allergic disease, which represented in hazard ratio and $95 \%$ confidence intervals (CI). Adjustments were made for potential confounders. Kaplan-Meier curves were generated to depict the development of outgrown of allergic diseases over time.

\section{Results}

Cumulative incidence of allergic diseases

Of the 369 mother-children pairs recruited in our cohort, 279 (75.6\%) has completed data collection and follow-up until 30 months of age. Demographic data is shown in Table 1. Fifty-five percent of the infants are male. Most of the participants has no family history of atopic diseases (74.2\%). Forty percent of infants were delivered by cesarean section. Forty-one percent of the infants were exclusively breastfed for at least four months.

The cumulative incidence of allergic diseases (Figure 1A) was $46.2 \%$, including AD (13.9\%, N=39), FA $(7.2 \%, \mathrm{~N}=20)$, ever wheezing $(24 \%, \mathrm{~N}=67)$, and chronic rhinitis $(18.6 \% \mathrm{~N}=52)$. If asthma was counted instead of ever wheezing and AR was counted instead of chronic rhinitis, the cumulative incidence of allergic diseases 
was $17.2 \%$; asthma $(0.7 \%, \mathrm{~N}=2), \mathrm{AR}(6.1 \%, \mathrm{~N}=17)$. $\mathrm{AD}$ and $\mathrm{FA}$ were the most prevalent allergic diseases during the first year of life. Most of the cases with $\mathrm{AD}$ and FA were outgrown overtime. Meanwhile, the prevalence of wheezing and rhinitis subsequently increased. The point prevalence of allergic diseases from birth up to 30 months is shown in Figure 1B.

Of 39 subjects with $\mathrm{AD}$, mild $\mathrm{AD}$ was the most observed $(74.4 \%, \mathrm{~N}=29)$ followed by moderate $\mathrm{AD}$ $(23.1 \%, \mathrm{~N}=9)$ and severe $\mathrm{AD}(2.5 \%, \mathrm{~N}=1)$. Of 20 subjects with $\mathrm{FA}, 13$ subjects $(65.0 \%)$ had IgE-mediated reactions. Five subjects $(25.0 \%)$ had non-IgE mediated reactions, including three subjects with allergic proctocolitis and two with FPIES. Another two subjects (10.0\%) with mixed-IgE mediated reactions, eczema was flared after oral food challenge to cow's milk. Hen's egg was the most common food allergen $(30.0 \%, \mathrm{~N}=6)$, followed by cow's milk $(25.0 \%, \mathrm{~N}=5)$, wheat $(20.0 \%, \mathrm{~N}=4)$, shrimp $(10.0 \%, \mathrm{~N}=2)$, banana $(10.0 \%, \mathrm{~N}=2)$ and fish $(5.0 \%, \mathrm{~N}=1)$. In all subjects with ever-wheezing $(\mathrm{N}=67)$, most had wheezing for only one time $(65.7 \%, \mathrm{~N}=44)$. Twenty-two percent of subjects $(\mathrm{N}=15)$ met the definition of recurrent wheezing. Asthma was diagnosed only in two subjects ( $0.7 \%$ of all population subjects). For subjects with rhinitis $(\mathrm{N}=52)$, the symptoms started between age 1-2 years old in most cases. Interestingly, $9.6 \%(\mathrm{~N}=5)$ of subjects started to have rhinitis symptoms before one year of age. Seventeen subjects (6.1\% of all study population) were fitted with the criteria of AR in our study.

Development of atopic march and co-occurrence of allergic diseases

One-third of the subjects developed at least two types of atopic manifestations at the age of 30 months. This included 25\% who developed two types of atopic manifestations (AD+FA, AD+rhinitis, $\mathrm{AD}+$ wheezing, FA+rhinitis, FA+wheezing, wheezing +rhinitis), $5 \%$ who developed three types of atopic manifestations (AD+wheezing +rhinitis, $\mathrm{AD}+\mathrm{FA}+$ wheezing, $\mathrm{AD}+\mathrm{FA}+$ rhinitis), and less than 1 percent $(0.9 \%)$ who developed all 4 atopic manifestations (AD+FA+wheezing+rhinitis). Sixty percent of subjects with AD and $85 \%$ of subjects with FA developed other atopic diseases. Only $9 \%$ of the subjects with wheezing and $9 \%$ of subjects with rhinitis had other preceeding atopic phenotypes, either AD or FA. These numbers are demonstrated in Figure 1A.

Besides, our cohort found a strong association between allergic diseases. Subjects with FA had an increased risk of having $\mathrm{AD}$ with a hazard ratio (HR) of 6.5 (95\% CI, 3.2-13.06; $p<0.0001$ ) while subjects with AD also had 8.5 folds increased risk of having FA; HR=8.51 (95\%CI, 3.52-20.56; $p<0.0001)$. Having rhinitis before 12 months of age associated with having FA, with HR of $5.53(95 \% \mathrm{CI}, 1.01-30.46 ; p=0.049)$. Allergic rhinitis also significantly associated with ever-wheezing with OR of $3.56(95 \% \mathrm{CI}, 1.01-12.56 ; p=0.049)$.

Aeroallergen sensitization

SPT to aeroallergens was performed in 269 subjects (96.4\%) of whom completed the follow-up. Of these subjects, $36.8 \%$ (99/269) were sensitized to at least one aeroallergen. Polysensitization was found in about half of the sensitized patients (44\%). Dermatophagoides pteronyssinus was the most common sensitized allergen (86.0\%), followed by Bermuda grass (23.0\%), cat hair (22.0\%) and American cockroach (22.0\%), German Cockroach (18.0\%), dog hair (12.0\%), and Johnson grass (6.0\%) respectively. Sensitization to molds was relatively low, including Aspergillus spp. (2.0\%) andCladosporium spp. (1.0\%). Aeroallergen sensitization between healthy subjects and ever-wheezing $(35.4 \%$ vs $35.8 \%)$ and between healthy subjects and rhinitis (36.1\% vs $32.7 \%$ ) were not significantly different. Having household furry pets (cats and dogs) or neighbourhood furry pets did not increase sensitization to pets in our cohort.

Factors associated with the development of allergic diseases and atopic march

Factors related to the development of allergic diseases are demonstrated in Table 2 to Table 4 . First of all, high family income significantly increased risk of AD with adjusted hazard ratio (aHR) of 3.71 (95\% CI, 1.31$10.45 ; p=0.013)$. Pet ownership, interestingly, lowered the risk of $\mathrm{AD}$, aHR $=0.29$ (95\% CI, $0.12-0.71 ; p=$ $0.006)$. Maternal history of atopic diseases significantly increased the risk of FA with HR of 3.39 (95\%CI, $1.34-8.62 ; p=0.01$ ), while paternal atopy, parental atopy, or sibling(s) with atopy did not increase risk of any allergic diseases. Receiving antibiotics before six months of age substantially higher the risk of FA, aHR 
$=3.63(95 \% \mathrm{CI}, 1.49=8.88 ; p=0.005$. Surprisingly, exclusive breastfeeding for 4 to 6 months and more than 6 months increased risk of FA with aHR of 2.92 (95\%CI, 1.16-7.39; $p=0.023)$ and aHR of 16.01 (95\%CI, $3.27-78.38 ; p=0.001)$. In contrast, duration of exclusive breastfeeding less than 4 months lowered the risk of FA with aHR of $0.22(95 \% \mathrm{CI}, 0.08-0.60 ; p=0.003)$.

For subjects with wheezing, only factors associated with ever-wheezing is demonstrated in Table 4. High family income significantly increased the risk of having ever-wheezing with the adjusted odds ratio (aOR) of $6.98(95 \%$ CI, $1.69-28.79 ; p=0.007)$. Living in shophouse lowered the risk of ever-wheezing with aOR of 0.3 $(95 \%$ CI, 0.1-0.91; $p=0.033)$. Infants who exposed to antibiotics before 24 and 30 months of age significantly increased the risk of ever-wheezing with aOR of 2.79 (95\%CI, $1.54-5.04 ; p=0.001)$ and 2.38 (95\%CI, 1.33$4.27 ; p=0.004$ ). Infants who exposed to antibiotics before 6 months of age significantly increased the risk of recurrent wheezing with OR of $5.46(95 \% \mathrm{CI}, 1.58,18.93 p<0.007)$. More frequent exposure to antibiotics further increased the risk of ever-wheezing and recurrent wheezing; aOR of 1.51 (95\%CI, 1.21-1.88; $p<0.001$ ) and OR of $1.8(95 \% \mathrm{CI}, 1.13,2.87 ; p=0.014)$ respectively. Subjects who were having first wheeze before one year of age were twelve times increasing risk of recurrent wheezing with OR of 12.3 (95\%CI, 2.49-60.5). For rhinitis, antibiotics exposure between 12 and 18 months of age significantly higher the risk; aOR of 2.41 $(95 \%$ CI, $1.01-5.74) ; p=0.046)$. The most common indication for antibiotics used at our cohort was respiratory tract infections. Different mode of delivery, gender, having older siblings, daycare attendance, regular moisturizer used during infancy period, smoke exposure, indoor pollution and aeroallergen sensitization did not significantly increase the risk of allergic manifestations in our cohort.

For atopic march development, having $>2$ atopic manifestations related to high family income $(\mathrm{HR}=10$ (95\%CI, 1.26-79.34; $p=0.029)$, maternal atopy (HR $=3.06(95 \% \mathrm{CI}, 1.09-8.58 ; p=0.034)$, having FA (HR $=21.19(95 \% \mathrm{CI}, 5.71-78.59 ; p=0.001)$, having $\mathrm{AD}(\mathrm{HR}=6.17(95 \% \mathrm{CI}, 2.7-14.13 ; p<0.001)$, having rhinitis $(\mathrm{HR}=3.83(95 \% \mathrm{CI}, 1.75-8.38 ; p=0.001)$, and multiple sensitization to aeroallergens $(\mathrm{HR}=5.33(95 \% \mathrm{CI}$, $1.43-19.94 ; p=0.013)$.

The outgrowing of allergic diseases

Forty-nine percent and $68.2 \%$ of subjects with $\mathrm{AD}$ were outgrown at the age of 12 months at age 24 months, regardless of severity, the onset of $\mathrm{AD}$ or food allergy status (Figure 4A). Meanwhile, $40 \%$ and $75 \%$ of FA was outgrown at age 12 months and 24 months, regardless of food allergens, the onset of FA or AD status (Figure 4B). Type of food allergic reaction did not significantly influence thr outgrown rate, only except the patients with allergic protocolitis from cow milk allergy whom HR of outgrown rate was 17.55 compared to others. All subjects with shrimp allergy were not outgrown. No factors could predict the outgrown of AD and FA in our cohort.

\section{Discussion}

This study is one of the only few allergy birth cohort conducting in developing Asian countries. The cumulative incidence of allergic diseases in early childhood in our population was comparable to studies in other countries ${ }^{6,18,19}$. The percentage of multiple allergic phenotypes was about the same rate as previous cross-sectional studies ${ }^{20,21}$. The contribution of both genetic and early-life environmental factors for the risk of allergic diseases was confirmed in our cohort. Maternal atopic disease, not paternal or siblings, was found to increase risk of FA and the development of atopic march. This suggests the prominent influence of maternal factors to atopic outcome than the general family history of atopy.

Similar to several previous studies ${ }^{22,23}$, our study has shown that early antibiotic exposure in infants was a predisposing factor of FA, wheezing and rhinitis. Antibiotics use in early life could modify gut microflora and subsequently promotes Th2-predominant allergic immune responses ${ }^{24}$. Strong associations were also found with multiple courses of antibiotic treatment. Families with high socioeconomic status supposed to have more hygienic lifestyles, leading to the less exposure to microorganisms and might relate to increase risk of allergies and atopic march development. Pet owner, on the contrary, protected for AD development in our population which is similar to the previous findings in German birth cohort study ${ }^{25}$. Taken together, factors related to hygiene hypothesis seems to be important on the development of allergy in our population. 
Breastfeeding is the preferrable method for infant nutrition. However, the protection against the development of allergic diseases is inconclusive. Recent meta-analysis showed the protective effect of prolonged breastfeeding on asthma in children aged 5-18 years, AR in children up to 2 years of age, AD in children up to 2 years of age $^{26}$. However, no association was found between breastfeeding and FA ${ }^{27}$. Our study found a negative effect of prolonged exclusive breastfeeding on the development of FA., even though the influence of reverse causality cannot entirely be rule out. As the parents with a family history of atopy might tend to breastfed the infants, with the prospect that breastfeeding might minimize the risk of allergy.

Other contributing factors to the development of allergic diseases such as delivery mode, gender, parental education, having siblings, daycare attendance, regular moisturizer used and smoke exposure ${ }^{3,4}$ were not significantly associated with allergic manifestations in our cohort. Interestingly, our findings are in line with some other studies such as GUSTO Singapore cohort ${ }^{28}$ and Avon Longitudinal Study of Parents and Children ${ }^{29}$, revealing that the cesarean section was not associated with allergic diseases. This fact is opposed to the finding of Norwegian birth cohort studies ${ }^{30}$. These findings, again, emphasized that data from other cohorts cannot directly be adopted to developing Asian populations.

There are many strengths of our study. First, the subjects were prospectively followed by allergy specialists. The diagnosis of allergy was done using the International criteria and supported by positive physical findings. Food allergy was confirmed by an oral food challenge test. All these reasons made our data highly reliable. Recall bias is minimized due to the frequent and regular visits. The limitation of our study is the small number cohort from a single center, which recruited only the healthy term subjects from the inner city area.

In conclusion, our study confirmed that allergic diseases are among the most common health diseases in young children, even in developing Asian country where the incidence of allergic diseases was previously perceived to be low. Natural course, risk and protective factors of allergic diseases and atopic march development were identified in the prospective birth cohort manner. Identify the populations who are at-risk and early modification of influence factors might promote immunologic tolerance and prevent the development of atopic march.

\section{Acknowledgements}

This study was supported by Ratchadapisek Research Funds (Grant No. CUGR(S)_61_38_30_03), Chulalongkorn University, Bankgok, Thailand and Thrasher Research Fund. The authors would like to thank Paediatric allergists and research team from Pediatric Allergy and Clinical Immunology Research Unit, Division of Allergy and Immunology, Department of Paediatrics, Faculty of Medicine, King Chulalongkorn Memorial Hospital, and Center of Excellence Clinical Virology, Faculty of Medicine, Chulalongkorn University, Bangkok, Thailand especially Ms. Wanlapa Weerapakorn, Ms. Donchida Srimuan, Ms. Siwaphon Chaimuk the research coordinators and Ms. Thaneeya Tongkaew our study nurse, for enrollment and followup the subjects in this cohort.

\section{References}

1. Asher MI, Montefort S, Bjorksten B, et al. Worldwide time trends in the prevalence of symptoms of asthma, allergic rhinoconjunctivitis, and eczema in childhood: ISAAC Phases One and Three repeat multicountry cross-sectional surveys. Lancet. 2006;368(9537):733-743.

2. Campbell D, Boyle R, Thornton C, Prescott S. Mechanisms of allergic disease-environmental and genetic determinants for the development of allergy. Clinical 85 Experimental Allergy. 2015;45(5):844-858.

3. McGowan EC, Bloomberg GR, Gergen PJ, et al. Influence of early-life exposures on food sensitization and food allergy in an inner-city birth cohort. Journal of Allergy and Clinical Immunology.2015;135(1):171-178. e174.

4. McBride D, Keil T, Grabenhenrich L, et al. The EuroPrevall birth cohort study on food allergy: baseline characteristics of 12,000 newborns and their families from nine European countries.Pediatric Allergy and Immunology. 2012;23(3):230-239. 
5. Loo EXL, Sim JZT, Goh A, et al. Predictors of allergen sensitization in Singapore children from birth to 3 years. Allergy, Asthma \& Clinical Immunology. 2016;12(1):1-7.

6. Kim HY, Kwon EB, Baek JH, et al. Prevalence and comorbidity of allergic diseases in preschool children. Korean journal of pediatrics. 2013;56(8):338.

7. Lee M-T, Wu C-C, Ou C-Y, et al. A prospective birth cohort study of different risk factors for development of allergic diseases in offspring of non-atopic parents. Oncotarget. 2017;8(7):10858.

8. Narita M, Qiu D, Hamaguchi M, et al. Maternal diet during pregnancy and wheeze and eczema in infants; the Japanese birth cohort (T-CHILD) study. Journal of Allergy and Clinical Immunology.2011;127(2):AB174.

9. Cochrane S, Beyer K, Clausen M, et al. Factors influencing the incidence and prevalence of food allergy. Allergy.2009;64(9):1246-1255.

10. Koplin JJ, Allen KJ, Gurrin LC, et al. The impact of family history of allergy on risk of food allergy: a population-based study of infants.International journal of environmental research and public health. 2013;10(11):5364-5377.

11. Liu C-A, Wang C-L, Chuang H, Ou C-Y, Hsu T-Y, Yang KD. Prenatal prediction of infant atopy by maternal but not paternal total IgE levels. Journal of allergy and clinical immunology.2003;112(5):899-904.

12. Lodge CJ, Tan D, Lau M, et al. Breastfeeding and asthma and allergies: a systematic review and meta-analysis. Acta Paediatrica. 2015;104:38-53.

13. Bloomfield SF, Rook GA, Scott EA, Shanahan F, Stanwell-Smith R, Turner P. Time to abandon the hygiene hypothesis: new perspectives on allergic disease, the human microbiome, infectious disease prevention and the role of targeted hygiene. Perspectives in public health.2016;136(4):213-224.

14. Aw M, Penn J, Gauvreau GM, Lima H, Sehmi R. Atopic March: Collegium Internationale Allergologicum Update 2020. International Archives of Allergy and Immunology. 2020;181(1):1-10.

15. Tsakok T, Marrs T, Mohsin M, et al. Does atopic dermatitis cause food allergy? A systematic review. Journal of Allergy and Clinical Immunology. 2016;137(4):1071-1078.

16. Shen Y, Zeng J-H, Hong S-L, Kang H-Y. Prevalence of allergic rhinitis comorbidity with asthma and asthma with allergic rhinitis in China: a meta-analysis. Asian Pac J Allergy Immunol. 2018.

17. Bantz SK, Zhu Z, Zheng T. The atopic march: progression from atopic dermatitis to allergic rhinitis and asthma. Journal of clinical \&5 cellular immunology. 2014;5(2).

18. Chen J, Hu Y, Allen KJ, Ho MH, Li H. The prevalence of food allergy in infants in Chongqing, China. Pediatric Allergy and Immunology.2011;22(4):356-360.

19. Laughter D, Istvan JA, Tofte SJ, Hanifin JM. The prevalence of atopic dermatitis in Oregon schoolchildren. Journal of the American Academy of Dermatology. 2000;43(4):649-655.

20. Somanunt S, Chinratanapisit S, Pacharn P, Visitsunthorn N, Jirapongsananuruk O. The natural history of atopic dermatitis and its association with Atopic March. Asian Pac J Allergy Immunol.2017;35(3):137-143.

21. Wananukul S, Chatproedprai S, Tempark T, Phuthongkam W, Chatchatee P. The natural course of childhood atopic dermatitis: a retrospective cohort study. Asian Pacific journal of allergy and immunology.2015;33(2).

22. Johnson CC, Ownby DR, Alford SH, et al. Antibiotic exposure in early infancy and risk for childhood atopy. Journal of Allergy and Clinical Immunology. 2005;115(6):1218-1224.

23. Thomas M, Custovic A, Woodcock A, Morris J, Simpson A, Murray CS. Atopic wheezing and early life antibiotic exposure: a nested case-control study. Pediatric allergy and immunology.2006;17(3):184-188. 
24. Bousquet J, Gern JE, Martinez FD, et al. Birth cohorts in asthma and allergic diseases: report of a NIAID/NHLBI/MeDALL joint workshop. Journal of Allergy and Clinical Immunology.2014;133(6):15351546 .

25. Zirngibl A, Franke K, Gehring U, et al. Exposure to pets and atopic dermatitis during the first two years of life. A cohort study.Pediatr Allergy Immunol. 2002;13(6):394-401.

26. Oddy WH. Breastfeeding, childhood asthma, and allergic disease.Annals of Nutrition and Metabolism. 2017;70(Suppl. 2):26-36.

27. Matsumoto N, Yorifuji T, Nakamura K, Ikeda M, Tsukahara H, Doi H. Breastfeeding and risk of food allergy: A nationwide birth cohort in Japan. Allergology International. 2020;69(1):91-97.

28. Loo EXL, Sim JZT, Loy SL, et al. Associations between caesarean delivery and allergic outcomes: Results from the GUSTO study.Annals of Allergy, Asthma 83 Immunology. 2017;118(5):636-638.

29. Maitra A, Sherriff A, Strachan D, Team AS, Henderson J. Mode of delivery is not associated with asthma or atopy in childhood. Clinical \& Experimental Allergy. 2004;34(9):1349-1355.

30. Magnus MC, Håberg SE, Stigum H, et al. Delivery by Cesarean section and early childhood respiratory symptoms and disorders: the Norwegian mother and child cohort study. American journal of epidemiology.2011;174(11):1275-1285.

\section{Figure legends}

Figure 1 : The cumulative incidence of allergic diseases (A) and point prevalence of allergic diseases (B) from birth up to 30 months.

Figure 2: Outgrown rate of atopic dermatitis (AD) (A) and food allergy (B).

\section{Hosted file}

Figure.pdf available at https://authorea.com/users/377336/articles/494048-natural-courseand-factors-associated-with-allergic-diseases-in-early-life-data-from-a-developingasian-country

\section{Hosted file}

Table.pdf available at https://authorea.com/users/377336/articles/494048-natural-course-andfactors-associated-with-allergic-diseases-in-early-life-data-from-a-developing-asiancountry 